\title{
Au-delà du bellicisme et du pacifisme : l'indifférentisme des avant-gardes
}

Jenseits von Bellzismus und Pazifismus: der Indifferentismus der Avantgarden Beyond bellicism and pacifism: the avant-gardes' indifferentism

\section{Thomas Keller}

\section{(2) OpenEdition}

\section{Journals}

Édition électronique

URL : http://journals.openedition.org/ceg/2064

DOI : $10.4000 /$ ceg. 2064

ISSN : 2605-8359

\section{Éditeur}

Presses Universitaires de Provence

\section{Édition imprimée}

Date de publication : 15 juin 2014

Pagination : 167-184

ISSN : 0751-4239

Référence électronique

Thomas Keller, «Au-delà du bellicisme et du pacifisme : l'indifférentisme des avant-gardes », Cahiers d'Études Germaniques [En ligne], 66 | 2014, mis en ligne le 17 décembre 2017, consulté le 10 décembre 2020. URL : http://journals.openedition.org/ceg/2064 ; DOI : https://doi.org/10.4000/ceg.2064 


\title{
Au-delà du bellicisme et du pacisfime - l'indifférentisme des avant-gardes
}

\author{
Thomas KELLER
}

Aix Marseille Université

\section{Introduction}

En 1929 Hannah Höch, femme dadaïste, peint un dessin aquarelle qui est énigmatique au premier abord ${ }^{1}$. Le personnage représenté est Salomo Friedlaender, alias Mynona, qui apparaît souvent dans les réalisations dadaïstes, par exemple dans le collage Schnitt mit dem Küchenmesser durch die erste Bierbauch-Kulturepoche Deutschlands (1919) de Höch ou dans les œuvres de Raoul Hausmann. Le philosophe quelque peu occulte du dadaïsme y est facilement identifiable à partir de sa silhouette, de la forme de sa tête et / ou grâce à une citation rappelant son œuvre Schöpferische Indifferenz / Indifférence créatrice. Le tableau de Höch expose le philosophe à l'appétit sexuel légendaire, en train d'écraser avec ses pattes d'éléphant le célèbre roman de Remarque Im Westen nichts Neues, paru en 1929. Sur la carte, les lettres du titre du roman, dont l'action se déroule surtout dans les tranchées en France, ne recouvrent que l'Allemagne. Le texte de Remarque n'est pas franco-allemand. On y regardant de plus près, le spectateur s'aperçoit que Höch met sous les pieds de Mynona un titre incomplet, amputé du mot « Nichts »: « IM WESTEN NEUES. » À l'Ouest du nouveau. Le texte de Remarque ne traite pas du Nichts, du rien. Le rien est un des concepts-clés du dadaïsme et notamment de l'indifférentisme. Il est créateur, non le contraire d'une information donnée. Ainsi est-il impossible de terminer une guerre par un pacifisme qui n'est que l'opposition au bellicisme. Le rien est une polarité tierce. C'est justement ce que Remarque n'aurait pas compris.

\footnotetext{
${ }^{1}$ Aquarelle reproduite in Detlef THIEL, « Einleitung. Stiche in Wespennester. Mynona dekonstruiert die Moderne (Remarque / Tucholsky) », in Salomo FRIEDLAENDER / MYNONA, " Hat Erich Maria Remarque wirklich gelebt ? Der Holzweg zurück », Detlef THIEL (éd.), Gesammelte Schriften, vol. 11, Hartmut GEERKEN, Detlef THIEL (éd.), Herrsching, Waitawhile Ed., 2010, p. 9-92, p. 37.
} 


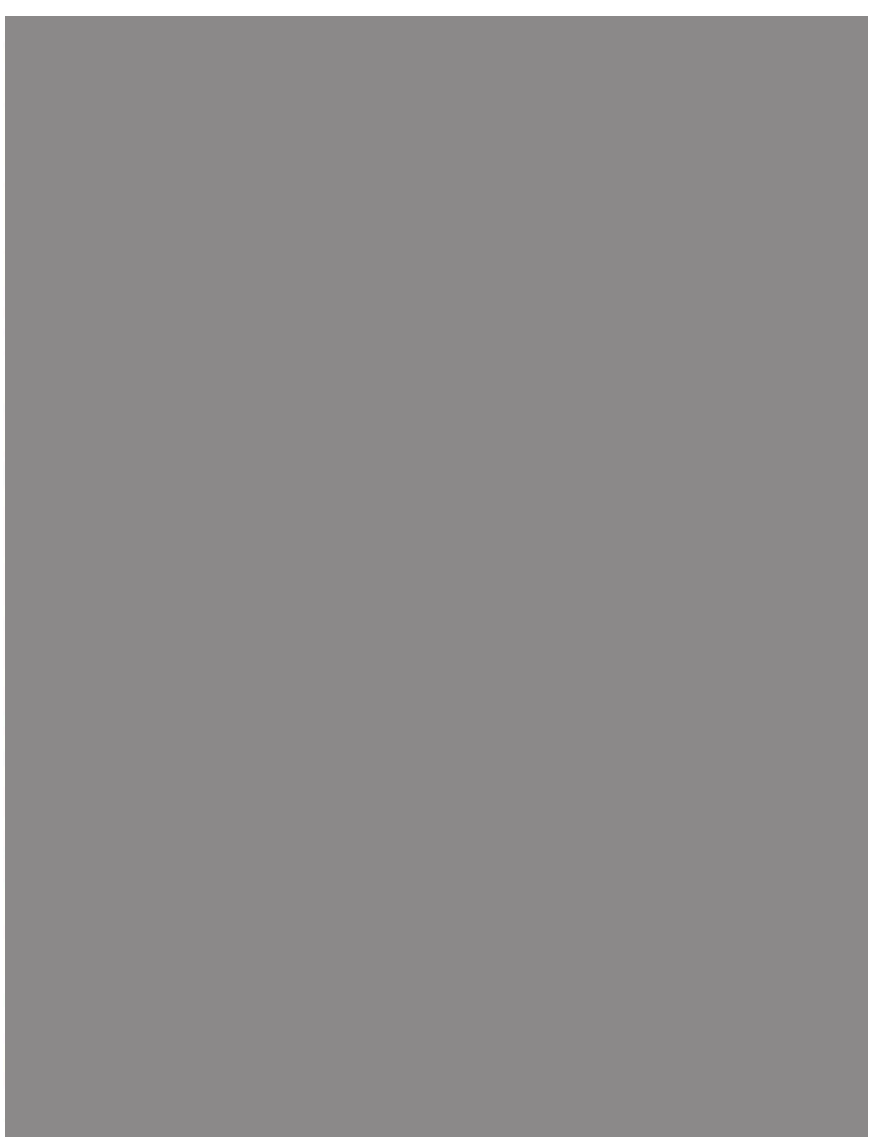

Hannah Höch, Der Gigant (Im Westen nichts Neues), Berlin 1929, AKB Kunstsammlung,

(C) ADAGP, Paris 2014

Salomo Friedlaender (né en 1871 à Gollantsch, près de Posen ; mort en 1946 à Paris) avait, dès la parution du bestseller Im Westen nichts Neues, élaboré une critique cinglante du roman. Dans Hat Erich Maria Remarque wirklich gelebt? Eine Denkmalsenthüllung, von Mynona (1929), Friedlaender a pris l'œuvre de Remarque comme cible puisqu'elle serait représentative d'une «médiocrité neutre » et correspondrait au " goût de Monsieur Toutlemonde ${ }^{2} »$. L'auteur n'aurait pas « le moindre talent ${ }^{3} »$. Le reproche

2 Salomo Friedlaender / MYNONA, Hat Erich Maria Remarque wirklich gelebt? Eine Denkmalsenthüllung, von Mynona (1929) ; je cite d'après Salomo FRIEDLAENDER / MYNONA, " Hat Erich Maria Remarque wirklich gelebt ? Der Holzweg zurück », p. 95. Je remercie vivement $M$. Thiel pour ses précieuses informations.

Voir aussi : «Remarques Werk reflektiert eine mittelmässige Gesamtverfassung so neutral, trifft damit so kräftig ins Herz des Allerweltgeschmacks, dass es sich zu meiner Zielscheibe am besten eignet» (MYNONA, Remarque, p. 93). 
central de Mynona vise le rapport qu'entretient le texte avec la guerre. Le roman est « neutre », c'est-à-dire obéit à une indifférence non-créatrice qui serait incapable de sortir de la logique meurtrière ${ }^{4}$.

Les invectives de Mynona provoquent une réplique sévère de Tucholsky ${ }^{5}$. S'il est vrai que l'éditeur Ullstein a imposé à Remarque des modifications destinées à produire un bestseller et a fait de l'auteur un simple soldat loin du milieu artistique ${ }^{6}$, les révélations de Mynona concernant les détails biographiques (par exemple la référence à la ville natale Osnabrück...) sont parfois gratuites. Aujourd'hui nous lisons les textes de Remarque et de Mynona en ayant connaissance des actions des nazis contre le film américain tourné d'après le roman. Au lieu de railler le pauvre Remarque sur des centaines de pages, Mynona aurait pu mieux utiliser son temps en prenant pour cible des auteurs bien plus douteux. Friedlaender a d'ailleurs payé son insolence impitoyable. Il fut très isolé pendant son exil en France à partir de 1933. Ainsi Thomas Mann a-t-il refusé son soutien pour le faire venir aux États-Unis après l'occupation de la France par la Wehrmacht.

L'analyse de Mynona mérite pourtant notre plus grand intérêt parce que l'indifférentisme représente une conception-clé des avant-gardes. Elle permet de retracer une position qui se situe au-delà du bellicisme et du pacifisme et de comprendre une nouvelle argumentation transculturelle. Le refus du pacifisme chez les dadaïstes allemands trouve son parallèle dans les avantgardes françaises. Les dadaïstes et futurs surréalistes en France ont peu d'estime pour Rolland ou pour Barbusse, l'auteur du Feu (1916). Les propos à ce sujet sont nombreux. Le plus connu est probablement le manifeste $L a$ Mobilisation contre la Guerre n'est pas la Paix, publié en juin 1933 à l'occasion du congrès international contre la guerre prévu à Genève : «Ainsi la bonne volonté évangélique des intellectuels de tous les partis trouve l'occasion, une fois de plus, de se manifester et d'exercer ses ravages sous prétexte de paix sur la terre ${ }^{7}$.»

3 « Remarque hat nicht das allergeringste Talent, dafür aber ist er Genius par excellence. "Der Mensch ist gut" [...] heisst ein Ausspruch. [...] Der nackte Mensch ist gut » (Mynona cite Leonhard Frank, TK : MYNONA, Remarque, p. 149).

${ }_{4}$ « Remarque, ein reproduktives Genie, hat der Menschheit den Weltkrieg friedlich reproduziert » (MYNONA, Remarque, p. 150).

5 « Die spezifisch deutsche Widerwärtigkeit, die die Luft unserer Politik so verpestet, weht durch dieses Buch Mynonas. Hierzulande werden Einwände damit erwidert, daß man sagt: der Einwendende habe einen roten Bart und eine verstopfte Schwiegermutter. Statt Breitscheid und Hilferding als geistige Typen zu verhöhnen und zu bekämpfen, wird argumentiert: "Und dann hat sich Breitscheid im Jahre 1897 eine Goldplombe machen lassen, aber nur für Amalgam bezahlt!" Anathema sit », Ignaz WROBEL (pseudonyme de Kurt TUCHOLSKY), « Hat Mynona wirklich gelebt? », Die Weltbühne, 31 décembre 1929, p. 15 sq.

${ }^{6}$ Günter OeSTERLE, « Das Kriegserlebnis im für und wider. "Im Westen nichts Neues" von Erich Maria Remarque (1929) », in Dirk VAN LAAK (dir.), Literatur, die Geschichte schrieb, Göttingen, Wallstein, 2011, p. 213-223.

${ }^{7}$ La Mobilisation contre la Guerre n'est pas la Paix, in Maurice NADEAU, Histoire du surréalisme, Paris, Seuil, 1964, p. 371-376. 
Les surréalistes nient toute force de conviction aux pacifistes, chez des écrivains comme Romain Rolland et Barbusse, ou chez Gandhi ${ }^{8}$. Leur antipacifisme est néanmoins différent de celui de Mynona. Il est dû en outre à une décision politique, à une prise de parti pour la cause révolutionnaire. Toutefois on peut y voir une critique commune de l'idéalisme, de la bonne volonté évangélique, de ce qu'on appelle l'angélisme en français ou Gutmenschentum en allemand. Ce refus s'exprime à travers une surenchère : le recours à la force est inévitable pour changer les bases des cultures belligènes. Peter Bürger ${ }^{9}$ et Karl Heinz Bohrer ${ }^{10}$ y perçoivent, de façon certes très différente, une violence esthétique. Rieuneau y voit le fruit d'un désespoir déclenchant la résistance révolutionnaire ${ }^{11}$. Ces interprétations désamorcent l'idolâtrie de la violence. Souligner l'ambiguité des dadaïstes et séparer les tendances cyniques préparant le fascisme des tendances « kyniques ${ }^{12}$ » (Sloterdijk) n'est guère satisfaisant non plus. L'approbation de la violence effective fait consensus pour ces avant-gardes ${ }^{13}$. J'y perçois plutôt une mobilisation qui suscite une autre force pour répondre aux atrocités de la machine de guerre déclenchées par les États-nations. La tentative de gagner

\footnotetext{
8 « Nous tenons à dénoncer, une fois de plus, le rôle néfaste et profondément contrerévolutionnaire des intellectuels qui en prennent l'initiative. Barbusse auteur de Jésus et Romain Rolland apologiste de Gandhi sont actuellement dans le monde les propagateurs les plus dangereux d'un mysticisme humanitaire plus pernicieux à tout prendre que n'importe quelle théologie abstraite.

L'idéalisme et la mystique de la non-violence sont les bases et les soutiens de tous les impérialismes, de toutes les oppressions.

En réponse au pacifisme officiel qui fait se muer les anges gardiens de la paix en ministres de la guerre ; en réponse à la plus vieille des formules impérialistes : " Si vous voulez la paix, préparez la guerre »; en réponse encore à l'hypocrite mot d'ordre de guerre à la guerre, nous disons : "SI VOUS VOULEZ LA PAIX, PRÉPAREZ LA GUERRE CIVILE ». ANDRÉ BRETON, ROGER CAILLOIS, RENÉ CHAR, RENÉ CREVEL, PAUL ELUARD, J.-M. MONNEROT, BENJAMIN PÉRET, GUI ROSEY, YVES TANGUY, ANDRÉ THIRION », in NADEAU, Histoire du surréalisme, p. 371-376, p. 376.

9 Peter BÜRGER, Der französische Surrealismus. Studien zur avantgardistischen Literatur. Um neue Studien erweiterte Ausgabe, Frankfurt am Main, Suhrkamp, 1996, p. 199.

${ }_{10}$ Karl Heinz BOHRER, Die Ästhetik des Schreckens. Die pessimistische Romantik und Ernst Jüngers Frühwerk, Frankfurt am Main/ Berlin/ Vienne, Ullstein, 1983, en particulier p. 325-412. Karl Heinz BOHRER, Plötzlichkeit. Zum Augenblick des ästhetischen Scheins, Frankfurt am Main, Suhrkamp, 1981.

${ }^{11}$ Maurice RIEUNEAU, Guerre et révolution dans le roman français de 1919 à 1939, (1 ${ }^{\text {re }}$ éd. Paris, Klincksieck 1974), Genève, Slatkine Reprints, 2000, p. 248.

12 Peter SLOTERDIJK, Kritik der zynischen Vernunft, Frankfurt am Main, Suhrkamp, 1983, vol. 2, p. 717. Sloterdijk caractérise le dadaïsme comme suit : «[E]inverstanden mit dem Schlimmsten » (p. 713), « unfreie Destruktivität» (p. 717), « Selbstmörder-Selbstbewusstsein » et « kalte Romantik» (p. 724).

${ }_{13}$ Je ne résiste pas à la tentation de rappeler le passage si souvent cité d'André Breton : « [L]'acte surréaliste le plus simple consiste, revolvers aux poings, à descendre dans la rue et tirer au hasard, tant qu'on peut, dans la foule », « Second Manifeste du Surréalisme », 1929, (André BReton, Manifestes du surréalisme, Paris, Gallimard (coll. «Idées »), 1963, p. 7-8; André BRETON, Euvres complètes, M. BONNET (éd.), Paris, Bibl. de la Pléiade, vol. I, 1988, 782 sq.) ; voir aussi Georges Bataille : «Le désespoir n'aboutit ni à la veulerie ni au rêve, mais à la violence » (Georges BATAILlE, Euvres complètes, t. I, Paris, Gallimard, 1987, p. 211).
} 
une position tierce reflète aussi une violence constitutive des avant-gardes et crée du même coup un lien entre les avant-gardes de pays différents ${ }^{14}$. L'indifférentisme est un concept transversal et transculturel qui permet de sortir de la binarité tout court, en particulier de la binarité guerre / paix et allemand / français. La violence fait du hasard l'élément tiers, le rien créateur d'un horizon nouveau. La notion d'indifférence ne doit pas être confondue avec une attitude désengagée. Elle relie deux significations et deux facultés : d'une part, elle décrit l'état d'esprit serein qui permet de s'affranchir de tout mécanisme polarisant; de l'autre, elle désigne l'absence d'antinomies telles que guerre et paix, ou français et allemand.

Je retracerai l'itinéraire du concept d'indifférence depuis le traité de Mynona jusqu'au procès Barrès à Paris. Je le décrirai en tant qu'il constitue un phénomène transitoire dans les rapports franco-allemands, puis $\mathrm{j}$ 'analyserai sa diversification en plusieurs figures : le dandy (Huelsenbeck), l'étranger (Tzara) et le témoin (Péret).

\section{L'indifférence créatrice et la guerre}

Le philosophe autodidacte Friedlaender publie son texte Schöpferische Indifferenz en 1918. En écrivant sous le pseudonyme Mynona - inversion d' « anonyme »-, il assume la posture qui supprime le nom au profit de sa multiplication. Il reprend des articles rédigés au cours de la guerre. Selon Friedlaender, l'indifférence créatrice réalise une "tendance indoaméricaine ", c'est-à-dire une attitude réconciliant la sérénité et la recherche du vide propres au bouddhisme avec le dynamisme du monde américain ${ }^{15}$.

Ce mysticisme de l'indifférence renoue aussi avec certaines caractéristiques de la pensée mystique européenne ; toutefois, Friedlaender critique aussi la formule de Nicolas de Cues de la coincidentia oppositorum qui débouche sur une identité des tendances contraires. Friedlaender, qui fut un temps le disciple du kantien non-conformiste Ernst Marcus, s'oppose ici à la conception kantienne de la vérité selon laquelle le Oui exclut le Non, selon laquelle une chose ne peut être à la fois son affirmation et son contraire. Friedlaender est convaincu de la possibilité tierce qui est neutre et créatrice.

\footnotetext{
14 Hanno Ehrlicher, Die Kunst der Zerstörung. Gewaltphantasien und Manifestationspraktiken europäischer Avantgarden, Berlin, Akademie Verlag, 2001.

${ }_{15}$ «Indo-Amerikanismus würde sich diese Tendenz bezeichnen; vom Innersten her alle Differenz, also Welt, Leib und Leben zu kultivieren. Hierdurch erst würde die Erde, deren Diametrik noch krampfhaft disproportioniert ist, die Erdkultur sich runden » (Salomo FRIEDLAENDER, Schöpferische Indifferenz, 1918, XXVII). Je cite d'après la nouvelle édition : Salomo FriedLAENDER / MYNONA, Schöpferische Indifferenz, Gesammelte Schriften, Vol. 10, Detlef THIEL (éd.), Waitawhile, Herrsching, 2009, p. 110. Voir aussi l'introduction instructive de Detlef THIEL, "Friedlaenders / Mynonas "Hauptwerk" ", ibid., p. 9-87.

Detlef THIEL, " "Philosophie ist keineswegs harmlos." Zum 150. Geburtstag von Ernst Marcus ", Kalonymos, Beiträge zur deutsch-jüdischen Geschichte aus dem Salomon Ludwig Institut, $9^{\mathrm{e}}$ année, 2006, $\mathrm{n}^{\circ} 4$, p. 1-4.
} 
Ce tiers devient la limite des différences. Nihil devient le nom personnel pour les noms infinis. Il s'agit d'une catégorie négative qui intègre les conflits et crée une nouvelle polarité. Friedlaender distingue la dualité néfaste de la tension créatrice. La sagesse du monde dépasse par exemple la polarité entre homme et femme ${ }^{16}$.

Il n'y a pas d'antisémites sans juifs ; il n'y a pas d'Allemands sans Français; il n'y a pas de guerre sans paix, en ce sens que l'un n'est pas le contraire de l'autre, c'est la relation entre les deux qui fait apparaître leur différence, celle entre guerre et paix mais aussi celle entre les belligérants ${ }^{17}$. Le troisième chapitre de Schöpferische Indifferenz est d'ailleurs intitulé «Frieden ${ }^{18} »$, « Paix ${ }^{19}$.

Indépendamment des formules parfois nébuleuses, Friedlaender tire les conséquences de l'impossibilité de combattre efficacement une chose par son contraire. Les deux combattants finissent toujours par se ressembler et par pérenniser le conflit. La simple contradiction n'échappe pas au mimétisme. L'invective contre Remarque dénonce l'incapacité de ce dernier d'explorer le terrain au-delà de la binarité entre guerre et paix, entre Allemand et Français. L'indifférentiste est avant tout la personne tierce qui refuse de prendre parti pour un pays, pour une culture et s'engage en faveur d'une rupture radicale.

Friedlaender transpose ainsi à la relation transculturelle l'objectif de l'avant-garde qui vise à abolir les cadres différenciateurs, afin de dépasser la différenciation sociale par la dédifférenciation. Dans son roman à clefs Graue Magie (1922) Mynona présente l'artiste Settegal (l'ami peintre de Mynona s'appelle Arthur Segal) et son invité le psychiatre Lemmis (lu à l'envers : Simmel, c'est-à-dire le psychiatre Ernst Simmel). Ils défendent le principe d'ôter et d'intégrer les cadres, qu'il s'agisse des concepts ou des tableaux. En cassant et en dissolvant les cadres de ses tableaux, Settegal ( c'est égal $\left.^{20} »\right)$ permet à l'œuvre d'interférer dans son environnement et de l'intégrer. La différenciation socio-culturelle - les distinctions entre art et vie, art et politique, privé et public, art et travail - disparaît. Cette indifférenciation devient alors transculturelle.

\footnotetext{
${ }^{16}$ « Weininger zum Beispiel zerschnitt den Magnetismus zwischen Mann und Weib, er bevorzugte das männliche Extrem als göttlich, verwarf das weibliche als teuflisch und missverstand also den Polarismus als den Dualismus. » (MYNONA, Schöpferische Indifferenz, p. 298).

${ }_{17}$ «Der Krieg zum Beispiel enthält in sich selber einen Gegensatz, etwa zwischen Deutsch und Englisch. Der Friede ist aber gar nicht Gegensatz zum Kriege, nicht dessen andrer Pol, sondern dessen Sinn, Seele, Individuum; das schöpferische Zentrum aller Diametrik. Der Friede bedeutet die Überwindung des Krieges, keineswegs im Sinne von Vernichtung, sondern von gleichsam musikalischer Beherrschung und Besiegung allen Widerstreits. » (ibid., p. 103).

${ }^{18}$ Ibid., p. 286-294.

19 Friedlaender y écrit : « Sein [des Schöpfers] inwendiger Frieden ist nicht der Funktionär des Weltkrieges, sondern des siegreichen Weltkrieges, der Weltharmonie, zu der jener immense Krieg nur die Einübung ist. », ibid., p. 292-293.

${ }^{20}$ Je remercie Detlef Thiel pour son décryptage.
} 
La stratégie avant-gardiste s'efforce de s'affranchir des différences à deux niveaux : celui des réalisations telles que le collage et la performance qui intègrent la vie quotidienne à l'art, dans un rapport transculturel; celui de l'indifférence créatrice, qui devient une puissance d'inversion, transformant la guerre en force qui s'anéantit elle-même. Le parallèle s'impose avec l'idée répandue en France de « der des ders » : la dernière des guerres, celle qui abolit la guerre en tant que moyen politique de régler les problèmes entre nations.

\section{Le dadandy}

Les dadaïstes décomposent l'indifférentisme en plusieurs stratégies. Hausmann et Höch, mais surtout Huelsenbeck rapprochent le pacifiste du bourgeois se réclamant de valeurs idéalistes, du Spiessbürger qui aime le confort, laisse les autres se battre et ne change rien au cours de l'histoire menant à des guerres entre nations. Ces auteurs prônent l'attitude dadaïste qui crée une distance permettant la mise en scène de rôles efficaces ${ }^{21}$. L'indifférence créatrice tout à la fois conserve et transforme les antagonismes, elle rend perméables les positions figées, ainsi que l'affirme Hausmann ${ }^{22}$

Huelsenbeck, qui fuit la guerre et devient cofondateur de Dada Zurich en 1916, reprend l'équivalence entre l'indifférentisme et l'indo-américanisme et l'intègre dans sa théorie de la simultanéité ${ }^{23}$. L'indo-américain est à la fois ancien et moderne. Il réussit à faire coexister les différences temporelles et spatiales. L'indifférentisme brise les murs séparant l'art de son environnement. La simultanéité et l'ubiquité insèrent l'héritage de l'indifférence dans le monde contemporain dynamique et bruyant. L'électricité transforme la nuit en jour, la télécommunication convertit la distance en présence.

${ }^{21}$ Cf. Bettina SCHASCHKE, Verwandlungskunst. Zum Verhältnis von Kritik und Selbstbehauptung in DADA, Berlin und Köln, Berlin, Gebr. Mann Verlag, 2004.

${ }^{22}$ « [...] Dada entspringt aus einer Einstellung, die man Schöpferische Indifferenz nennt: dort, wo die eigentlichen Gedankenbildungen anfangen und von wo aus man sie nie mehr in falsche Bahnen zurücklenken sollte und dürfte (Raoul HAUSMANN, cité d'après Detlef THIEL, « Einleitung », in MyNONA, Schöpferische Indifferenz, p. 38). Cf. Patrick LHOT, L'indifférence créatrice de Raoul Hausmann. Aux sources du dadaïsme, Presses universitaires de Provence, Aix-en-Provence, 2012. Je n'analyse pas ici le rôle de Hausmann pour le transfert culturel franco-allemand. Il transmet les théories d'Ernst Marcus et de Mynona surtout pendant son exil en France.

23 « Der Dadaist hat sich primitive Symbole für das Problem der Bewegung in dem 'Bruitismus' und in der 'Simultaneität' geschaffen - er ist der eigentliche Indoamerikaner, von dem S. Friedländer in seiner schöpferischen Indifferenz spricht. » (Richard HuELSENBECK, « Was ist Dadaismus und was will er in Deutschland? » in ID., En avant Dada, Hannover, Paul Steegemann Verlag, 1920, p. 34-44, p. 37, ainsi que dans Die dadaistische Bewegung. Eine Selbstbiographie [1920], cité par THIEL, «Einleitung », p. 39). 
À l'instar de Friedlaender, Huelsenbeck définit Dada « comme point d'indifférence entre contenu et forme, femme et homme, matière et esprit ». Dada est « la pointe d'un triangle qui s'élève au-dessus de la polarité linéaire des choses et des concepts humains ${ }^{24} »$. C'est ce rien qui crée une différenciation à un autre niveau ${ }^{25}$.

L'indifférentisme abolit le seuil entre le sacré et le profane. Il émerveille le monde ${ }^{26}$. Or Huelsenbeck relie le rien de l'indifférence et la guerre. Seul le rien permet d'en finir avec la guerre ${ }^{27}$. Il permet de transcender l'opposition entre guerre et paix $^{28}$. Cette formule revient plusieurs fois, dont la première dans un poème de 1916. Il traduit ce geste "cool" que Lethen décrit si bien dans sa Verhaltenslehre der Kälte. Huelsenbeck devient dadandy. Toutefois, cette posture n'est pas l'apanage des Allemands.

L'indifférentisme revêt ici les caractéristiques de l'acte gratuit, un acte sans aucune utilité, ni aucun intérêt, qu'Arthur Cravan et Felix Paul Greve affichent déjà avant 1914. Ces personnages scandaleux transcendent toutes les frontières entre les nations. Cravan, neveu d'Oscar Wilde, suisse et anglais, boxeur et artiste, brouille les pistes en Europe avant de disparaitre au Mexique. Le mythomane Greve, qui joue avec le crime, se réinvente une vie et une biographie et devient le célèbre écrivain canadien Frederick Philip Grove. Gide ${ }^{29}$, qui a reçu la visite de Greve et de Cravan, s'inspire de leur attitude et de leur disposition à aller jusqu'au meurtre sans motif dans la figure de Lafcadio, le protagoniste déconcertant et scandaleux qu'il met en scène dans le roman Les caves du Vatican. Il est impossible de déterminer son

\footnotetext{
${ }^{24}$ « Dada ist der Indifferenzpunkt zwischen Inhalt und Form, Weib und Mann, Materie und Geist, indem es die Spitze des magischen Dreiecks ist, das sich über die lineare Polarität der menschlichen Dinge und Begriffe erhebt ». (Richard HUELSENBECK, «Einleitung », in ID. (éd.), Dada-Almanach, Berlin, Erich Reiss Verlag, 1920, p. 3-9, p. 4.

${ }^{25}$ « Das Nichts ist kein Extrem, es ist auch bei Leibe nicht die Versöhnung seiner Extreme: ja es ist etwas polar Anderes als Nein. Das Nichts ist also gleichsam ihre magnetische Indifferenz, ihre polarisierende Neutralisation, ihre differenzierende Zentrierung, das Zentrum ihrer Antipodie. " (Richard HuELSENBECK, cité d'après Hanno Ehrlicher, Die Kunst der Zerstörung. Gewaltphantasien und Manifestationspraktiken europäischer Avantgarden, Berlin, Akademie Verlag, 2001, p. 196).

${ }^{26}$ « Dada repräsentiert jenen von S. Friedlaender propagierten Indoamerikanismus, wonach Heiligstes im Profansten und umgekehrt Profanstes im Heiligsten enthalten ist » (Richard HuELSENBECK, cité d'après THIEL, « Einleitung », Schöpferische Indifferenz, p. 39).

27 « Dies ist das bedeutende Nichts [...] Wir wollen die Welt mit Nichts ändern [...] wir wollen den Krieg mit Nichts zuende bringen » (Richard HuELSENBECK, «Erklärung » [vorgetragen im Frühjahr 1916 im Cabaret Voltaire], in Dada, Eine literarische Dokumentation, Reinbek bei Hamburg, Rowohlt, 1984, p. 34).

${ }_{28}$ « Dada ist die amerikanische Seite des Buddhismus [...] Ich halte den Krieg und den Frieden in meiner Toga, aber ich entscheide mich für den Cherry-Brandy-Flip. » (Richard HUELSENBECK, «Einleitung », in Dada-Almanach, 1920, p. 4).

${ }^{29}$ Cf. Iris Roebling, Acte gratuit. Variationen einer Denkfigur von André Gide, München, Fink, 2009
} 
« identité ». Il change constamment d'aspect et est partout à l'aise. À la fin de la « sotie » de Gide, les clairons annoncent la guerre ${ }^{30}$.

Le Westphalien Huelsenbeck, qui anime le dadaïsme à Berlin à partir de 1917, reste en contact avec le dadaïsme de langue française, voyage en Asie et deviendra plus tard aux États-Unis le psychanalyste Charles S. Hulbeck. Il s'installera dans le Tessin en 1970. S'il se situe dans la ligne de la posture transgressive et froide, il incarne aussi le magicien capable de ré-enchanter le monde et de se glisser dans des contextes culturels différents. La vie protéiforme s'oppose à l'intériorité si chère aux expressionnistes. Le moment vécu pleinement acquiert une valeur qui fait défaut à la culture traditionnelle. Le dandysme dadaïste met la vie en jeu et en danger tout en craignant d'être surpris par l'humiliation et le ridicule. Le dadandy ne veut pas perdre la face. Il n'admet aucune inféodation. Il combat la guerre en tant que guerre entre les nations. L'héroïsme à l'allemande ou à la française est dérisoire, mais le pacifisme l'est également. S'il est vrai que le dadaïste adhère à la violence esthétique, cette violence n'est pas purement esthétique ${ }^{31}$.

Huelsenbeck voit le côté américain du bouddhisme incarné dans le délinquant sexuel Alton ou dans l'imposteur Manolescu. Le dadandy flirte avec le crime. Il contribue à inaugurer un nouveau type social transfrontalier. Il se prolonge dans la tendance à la violence qui apparaît parfois dans le milieu libertaire et hédoniste des situationnistes et des spontanéistes. Il déplace et radicalise la formule de l'indifférence créatrice. L'indifférentiste devient ici une figure réprimant sa vulnérabilité et son empathie. Le chevalier-dandy, dont l'épée divise, déclame : "distinguo, ergo sum ». Je tranche, donc je suis. Je décide, donc je suis. Il hérite du code aristocratique en réservant son respect aux personnes dignes d'un duel («satisfaktionsfähig »). Toutefois il se décide pour un tiers, qui n'est ni pour la guerre, ni pour la paix, mais pour le Cherry-Brandy-Flip, le faisant ainsi glisser à un niveau supérieur. Ces distinctions violentes ne séparent ni le vrai du faux, ni le bon du mauvais, ni le beau du laid. Elles créent un maximum de différences engendrées par le dégoût pour des ressentiments répandus dans le monde bourgeois et nourris par les nationalismes.

Dans la mesure où l'indifférentisme légitime cette attitude de froideur, il n'est pas uniquement responsable de la scission entre les dadandys du type

\footnotetext{
${ }^{30}$ Gide a largement pratiqué la retenue et le silence pendant la guerre. Il devient à la fois cible et collaborateur des dadaïstes français rassemblés dans la revue Littérature (1919-1921). Voir le poème d'André BrETON « Pour Lafcadio», Dada, n 4-5, mai 1919, p. 12 : « Jamais je ne gagnerai tant de guerres / Des combattants ». Breton rappelle qu'aucune guerre n'est terminée tant que deux combattants s'affrontent.

${ }^{31}$ Wir waren gegen die Pazifisten, weil der Krieg uns die Möglichkeit gegeben hatte, überhaupt in unserer ganzen Gloria zu existieren. Und damals waren die Pazifisten noch anständiger wie heute, wo jeder dumme Junge mit seinen Büchern gegen die Zeit die Konjunktur nutzen will. Wir waren für den Krieg, und der Dadaismus ist noch heute für den Krieg. Die Dinge müssen sich stossen: es geht noch nicht grausam genug zu. » (Richard HUELSENBECK, «Erste Dada-Rede in Deutschland (février 1918), Document 4, in DadaBerlin, Texte, Manifeste, Aktionen, Karl RIHA (éd.), Stuttgart, Reclam, 1977, p. 17).
} 
Huelsenbeck et les dadaïstes militants et plus politisés d'Allemagne (Grosz et Heartfield). Il entraîne également la rupture avec les avant-gardistes plus compatissants. L'indifférentisme a aussi contribué à creuser l'écart entre les dadaïstes et les futurs surréalistes en France : cette dernière division se manifeste lors du procès Barrès quand les futurs surréalistes semblent maintenir contre l'indifférentisme dadaïste un jugement de valeur. Cette attitude attribue pourtant à l'indifférentisme un sens réduit, celui de flegmatisme, de fatalisme, de tiédeur du coeur. Il fait l'impasse sur sa charge émotionnelle et sa force mobilisatrice et créatrice qui comprend justement la violence.

En revanche, la lecture transculturelle permet de relier la question de la violence à ce "rien ", à ce choc dédifférenciateur qui met fin aux guerres connues. L'indifférentisme se diversifie et devient une passerelle entre cultures. S'il peut signifier la prise de distance et la froideur parfois communes aux dadaïstes et aux surréalistes, il peut aussi être plus compatissant. Toutefois il passe toujours par un acte violent dans lequel le transfert culturel franco-allemand fait de l'expérience de la guerre un médiateur pour sortir de la confrontation duelliste.

\section{Le lien transculturel et l'étranger}

L'indifférentisme signifie aussi la simultanéité et l'ubiquité de sons, de langues et de cultures. Dans le "poème simultan L'amiral cherche une maison à louer, poème par R. Huelsenbeck, M. Janko, Tr. Tzara », mis en scène en 1916 à Zurich, le rien représente le carrefour des cultures.

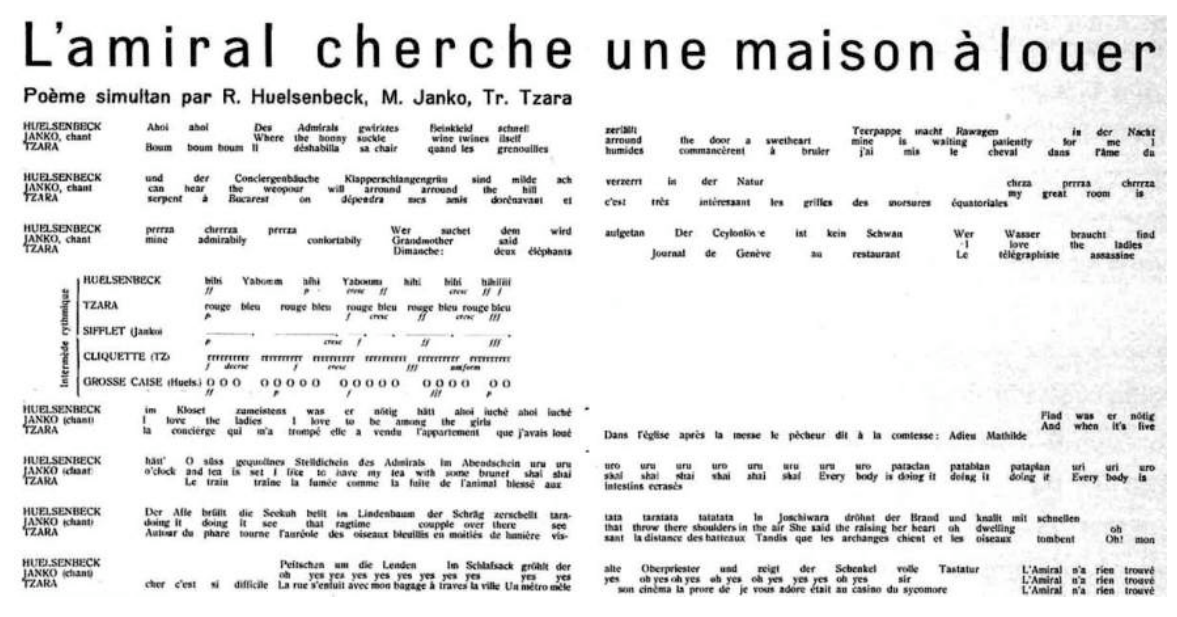

La nouvelle esthétique prend une forme concrète. Huelsenbeck déclame en allemand, Janko chante en anglais et Tzara parle en français. Les différentes voix ne représentent pas un seul texte traduit en plusieurs langues. 
Un chaos règne rappelant la guerre, la Babel des langues et des cultures. Ce n'est qu'à la fin que les trois s'exclament en chœur dans la même langue : «L'Amiral n'a rien trouvé ». Le « rien » est ici l'élément indifférenciateur qui permet de créer de nouvelles différences. Il s'est imposé. Les trois voix séparées dans les langues des trois parties belligérantes confluent et acquiescent à l'unisson et indépendamment de l'appartenance nationale. Tous accostent par ce rien qui les relie. Le français qui leur est commun est une langue étrangère pour les trois.

Huelsenbeck, Janko et Tzara participent à une performance qui dépasse les conflits entre nations. Le groupuscule pratique le multilinguisme. Les numéros 3 et 4 de Dada sont édités dans une version allemande et française. Le numéro 4-5 (1919) dissout plus que toute autre publication dadaïste les différences et pratique le bilinguisme : à côté de textes français de Tzara, Breton, Soupault, figurent des textes en allemand d'Arp, Serner, Huelsenbeck, Hausmann.

Huelsenbeck a transplanté les concepts de Mynona et les a recontextualisés dans un environnement plurinational, ou plutôt dans un environnement défiant toute nationalité. L'indifférentisme transforme ainsi la violence. Tandis que Huelsenbeck conserve largement la pose du dandy froid, l'indifférentisme transplanté prend chez Tzara une autre tournure ${ }^{32}$. En 1919, donc deux ans après le départ de Huelsenbeck de Zurich, Tzara présente dans la revue Dada «R. Huelsenbeck, Phantastische Gebete, Zurich». La caractérisation de Huelsenbeck par Tzara vaut davantage pour Tzara luimême : "Les langues différentes à son aide, - ses compagnons ses témoins. Il jette sa vision du paradis en enfer et réciproquement ; il n'y a rien de saint, tout est d'essence divine $»^{33}$. Pour caractériser Tzara, Henri Meschonnic parle d'une "éthique» cosmique, d'une "violence visible contre une violence invisible ${ }^{34} »$.

Le nom Tristan Tzara, pseudonyme de Samuel Rosenstock, originaire d'une famille juive de Roumanie, signale une référence à la tradition européenne du romantisme noir symbolisée dans le prénom Tristan et une extraterritorialité conférée par le mot roumain Tzara, qui signifie Terre. Tzara n'est ni français ni allemand. C'est un tiers. S'il décide d'écrire en français,

\footnotetext{
${ }^{32}$ Hubert Van Den Berg, Avantgarde und Anarchismus. Dada in Zürich und Berlin, Heidelberg, C. Winter, 1999.

ID., « Tristan Tzara's Manifest Dada 1918: Anti-Manifest oder manifestierte Indifferenz? Salomo Friedlaenders schöpferische Indifferenz und das dadaistische Selbstverständnis », Neophilologus, $\mathrm{n}^{\circ}$ 79, 1995, p. 353-376.

Wolfgang ASHOLT, «Internationale Strategien in futuristischen, dadaistischen und surrealistischen Manifesten », in Hubert VAN DEN BERG, Ralf GRÜTTEMEIER (dir.), Manifeste: Intentionalität, Amsterdam, Rodopi, 1998, p. 39-56.

${ }^{33}$ Dada, $\mathrm{n}^{\circ} 4-5$, mai 1919 , p. 27, revue rééditée dans Tristan TZARA, « Note sur Huelsenbeck ", in Dada est tatou. Tout est dada, Henri BÉHAR (dir.), Paris, GarnierFlammarion, 1996, p. 250.

${ }^{34}$ Henri MeschonNic, « Dada, on ne joue plus », Résonance Générale, ${ }^{\mathrm{er}}$ septembre 2009 (Revue-resonancegenerale.blogspotcom).
} 
cette langue ne devient jamais sa langue maternelle. Il est indifférent puisqu'il n'incarne pas les différences qui se combattent. Son dégoût dadaïste renoue autrement avec la « religion d'indifférence quasi-bouddhique». Son « À quoi bon », son « je m'en foutisme » ne l'empêchent pas d'exprimer dans son manifeste de 1918 : «Pas de pitié. Après le carnage l'espoir d'une humanité purifiée $»^{35}$. Tzara relie le rejet de la folie guerrière à l'œuvre rédemptrice de l'indifférentisme.

J'écris un manifeste et je ne veux rien [...] J'écris ce manifeste pour montrer qu'on peut faire les actions opposées ensemble. [...]

DADA NE SIGNIFIE RIEN

$[\ldots]$

Ordre $=$ désordre $;$ non $=$ non-moi $;$ affirmation $=$ négation $[\ldots]$

[I]1 y a un grand travail destructif, négatif, à accomplir. Balayer, nettoyer. La propreté de l'individu s'affirme après l'état de folie, de la folie agressive, complète, d'un monde laissé entre les mains des bandits qui déchirent et détruisent les siècles $[\ldots]$

DADA, hurlement des douleurs crispées, entrelacement des contraires, et de toutes les contradictions [...] LA VIE. ${ }^{36}$

La violence est positive en ce qu'elle débouche sur cette indifférence qui peut penser oui $=$ non. Pendant sa tournée en Allemagne en 1922, Tzara se réclame de la "religion d'indifférence », qui est violente sans faire le jeu des forces destructrices opposées dans les guerres :

Dada n'est pas du tout moderne, c'est plutôt le retour à une religion d'indifférence quasi-bouddhique [...]. Vous direz que cela est un paradoxe parce que Dada se manifeste par des actes violents. Oui, les réactions des individus contaminés par la destruction sont assez violentes, mais ces réactions épuisées, annihilées par l'instance satanique d'un «à quoi bon » continuel et progressif, ce qui reste et domine est l'indifférence. ${ }^{37}$

Bien que Tzara incarne un maillon crucial entre les contextes allemand et français, il ne relie pas en lui l'allemand et le français. Même après son arrivée à Paris en 1920, il garde son accent; il n'est, ni l'un, ni l'autre. Il témoigne des tiers qui sont plutôt victimes des antagonismes francoallemands. Il n'appartient à personne et à rien. S'il démystifie les croyances du monde bourgeois, il se réclame d'un mysticisme de l'immanence. Tzara identifie les similitudes des belligérants et celles des victimes. Il dit «non distinguo, ergo sum ». Il est l'indifférentiste du ni, ni. Son dégoût ne connaît pas la haine. L'étranger sombre paye de sa personne. Tout comme l'étranger

\footnotetext{
35 Tristan TZARA, « Manifeste Dada 1918 », dans Dada, n 3, décembre 1918, sans pagination.

${ }^{36}$ Ibid.

37 Tristan TZARA, « Conférence sur Dada, prononcée à Weimar et à Iéna en septembre 1922 », in ID., Dada est tatou. Tout est dada, Henri BÉHAR (éd.), Paris, Garnier-Flammarion, 1996, p. 267, ou Tristan TZARA, Euvres Complètes, t. 1, p. 419-424, p. 420.
} 
décrit par Simmel, il est dedans et dehors, il réunit et incarne l'appartenance et la non-appartenance. Il ne connaît pas de frontières.

L'indifférentisme, l'élément transculturel migrant entre 1916 et 1918 de Dada Zurich à Dada Berlin, puis à Dada Paris, s'incruste dans le contexte français avant d'être récusé par les futurs surréalistes qui épousent l'engagement politique et prennent parti. La revue Littérature garde pendant un moment des traces transculturelles. Walter Serner, le seul parmi les dadaïstes germanophones à y publier, figure parmi les éditeurs ; néanmoins, son texte est traduit en français. L'indifférentisme réapparaît recontextualisé par les avant-gardistes de langue française qui se revendiquent du dadaïsme. En 1920, Paul Éluard écrit dans Cinq moyens de pénurie Dada ou deux mots d'explications : «MORALE : Nous voyons tout, nous n'aimons rien, / Nous sommes indifférents, / In-diffé-rents. Nous sommes morts. Mais nous ne pourrissons $\operatorname{pas}^{38} \ldots$ » Dans le même numéro de Littérature, Aragon se réclame du rien : "plus de peintres, plus de littérateurs, plus de musiciens [...], plus de politiques, plus de prolétaires, [...] plus d'armée, plus de police, plus de patries [...], plus rien, plus rien, rien, RIEN, RIEN, RIEN ${ }^{39}$. »

Les morts vivants organisent un an plus tard le procès Barrès, qui représente une nouvelle manifestation de l'indifférentisme et introduit, grâce à une performance, la figure du témoin.

\section{Le témoin du procès Barrès ou le mutilé de guerre des avant-gardes}

Le procès Barrès a lieu le 13 mai 1921 à Paris. L'année précédente, l'État français a inventé la cérémonie du soldat inconnu. Une tombe a été installée sous l'Arc de Triomphe. On y transféré les restes de soldats non identifiés mais reconnus comme français. Huit corps sont exhumés. Le 11 novembre 1920, jour anniversaire de l'armistice, on commémore pour la première fois la mort pour la patrie. Le procès Barrès est une contre-performance avec un soldat inconnu mort-vivant.

André Breton est nommé Président. Théodore Fraenkel et Pierre Deval sont ses deux assesseurs. Georges Ribemont-Dessaignes assume le rôle de l'accusateur public. Louis Aragon et Philippe Soupault se déclarent prêts à défendre Barrès. Le 7 mai, la commission d'enquête décide d'accuser Maurice Barrès d' « attentat à la sûreté de l'esprit ». Selon le texte paru dans Littérature $^{40}$, Barrès a quitté Paris pour Metz, puis pour Aix-en-Provence où il tient un discours sur le sens de la guerre. Pendant le procès, Barrès est présent sous forme de poupée.

\footnotetext{
38 Paul ÉLUARD, « Cinq moyens de pénurie Dada ou deux mots d'explications », Littérature, $\mathrm{n}^{\circ} 13,1920$, p. 20.

39 Louis ARAGON, « Manifeste », Littérature, $n^{\circ} 13-14$, mai 1920, p. 1

40 «L'affaire Barrès I. Acte d'accusation - Témoignages », Littérature, n 20, août 1921, p. 1-24.
} 
Tandis que le président, l'accusateur et les défenseurs sont tous français, les sept témoins sont mixtes : Serge Romoff est russe ; Guiseppe Ungaretti italien ; Tristan Tzara roumain ; Rachilde, Drieu La Rochelle, Jacques Rigaut et Benjamin Péret français. Seuls Drieu et Ungaretti ont combattu dans les tranchées. Péret a été infirmier pendant, c'est-à-dire la dernière phase de la guerre qui doit être la « Der des Der ». Les témoins semblent représenter les pays en guerre, mais la mise en scène subvertit la fonction représentative. Parmi les témoins, trois ont un accent. Toutefois, aucun Allemand n'est présent. Cependant Péret, un Français, parle allemand en tant que «lieu tenant ». La notion de témoin prend une signification particulière dans ce contexte $^{41}$. Faire appel, dans le procès contre Barrès, à des déclarations de personnes étrangères signifie faire dépendre le jugement d'un savoir extérieur à la nation française. Le témoin sait quelque chose, que président et accusateur ignorent. Il transmet, mais il incarne aussi ce savoir ${ }^{42}$. La voix et le contact établi par les yeux contraignent les témoins à la véracité. Témoigner a donc deux significations : les propos des témoins promettent de dire la vérité ; ils témoignent physiquement. La vérité dont ils témoignent est justement un indice d'étrangeté. Leur prestige, leur crédit viennent de cette étrangeté. Les témoins ébranlent le rôle du président, de l'accusateur et de la défense, c'està-dire du mécanisme judiciaire binaire, au profit d'un tiers étranger.

Les témoins créent un lien physique avec les événements. Le premier témoin, Serge Romoff, représente l'esprit révolutionnaire. Contrairement au "nationalisme constructif " de la révolution russe, Barrès prêcherait un nationalisme odieux : " Le nationalisme de Barrès est destructif en tant qu'il entretient surtout la haine entre les nations. C'est un nationalisme offensif ${ }^{43}$. »

Tristan Tzara partage l'opinion négative sur Barrès. Mais il récuse la dualité séparant l'accusé coupable et les accusateurs innocents représentant le droit. Il conteste l'autorité du tribunal :

\begin{abstract}
Maurice Barrès est pour moi l'homme le plus antipathique que j'aie rencontré dans ma carrière littéraire ; c'est la plus grande fripouille que j'aie rencontré dans ma carrière poétique ; le plus grand cochon que j'aie rencontré dans ma carrière politique ; la plus grande canaille qui s'est produite en Europe depuis Napoléon. Je n'ai aucune confiance dans la justice, même si cette justice est faite par Dada. Vous conviendrez avec moi, M. le Président, que nous ne sommes tous qu'une bande de salauds et que par conséquent les petites différences, salauds plus grands ou salauds plus petits, n'ont aucune importance. ${ }^{44}$
\end{abstract}

Tzara renonce au jugement de l'un par l'autre. En cela, il finit la guerre. Il constate clairement la convergence entre le nationalisme de Barrès et le nationalisme allemand. Il rappelle le rejet de l'expressionisme allemand par

\footnotetext{
${ }^{41}$ «Einleitung », in Sibylle SCHMIDT, Sybille KrÄMER, Ramon VogES (dir.), Politik der Zeugenschaft. Zur Kritik einer Wissenspraxis, Bielefeld, transcript Verlag, 2010, p. 7-22.

${ }_{42}$ Cf. Sybille KräMER, Medium, Bote, Übertragung. Kleine Metaphysik der Medialität, Frankfurt am Main, Suhrkamp, 2008, p. 223-260.

${ }^{43}$ Littérature, ${ }^{\circ} 20$, p. 9.

${ }^{44}$ Ibid., p. 14.
} 
l'Action française, mais il est tout autant dégoûté par les petit-bourgeois admirateurs de Wagner et consommateurs de bière, par la «Bierbauchkultur » dénoncée dans le célèbre collage de Hannah Höch. Le wagnérisme relie les hommes politiques des puissances victorieuses et les nationalistes allemands :

\begin{abstract}
L'amour, tel que Barrès l'entend, est une vague rêverie allemande. Je le connais trop bien pour en être assez dégoûté. L'action que Barrès nous propose n'a réussi qu'à couvrir de matière fécale les toiles des peintres expressionnistes allemands. L'hypocrisie qui se cache derrière chaque phrase de Barrès est la même que celle d'un M. Hellfferich, Lloyd George, Briand ou Harding. Le wagnérisme a gonflé d'air les ventres des Allemands qu'on croit maintenant remplis de bière. Le wagnérisme est la caractéristique de l'œuvre de Barrès, qui est pompeuse et bombastique. $^{45}$
\end{abstract}

Tzara devient en quelque sorte " un témoin à décharge ", " de la même façon que Barrès est un témoin à décharge dans le procès du crétinisme européen ». Le tribunal n'est pas le moyen approprié pour sortir des dualismes néfastes. Tzara ne se range pas du côté des cultures antagonistes qui se ressemblent. Sa perception vient de l'extérieur.

Guiseppe Ungaretti, le futur compagnon de route de Mussolini, assume le point de vue indifférentiste :

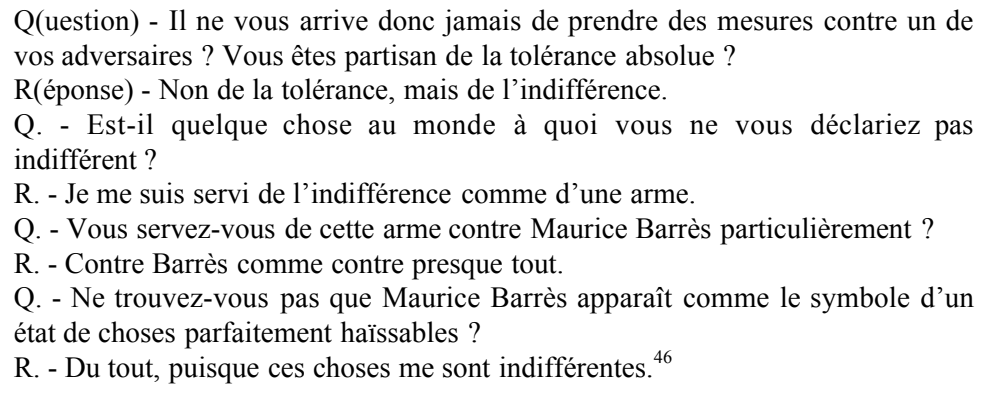

C'est lui le personnage le plus "froid" parmi les témoins, qui retient de l'indifférentisme surtout sa valeur tranchante. Il devient une arme - qui combat cependant pour un tiers.

Drieu La Rochelle, futur fasciste, rappelle le passé révolutionnaire de Barrès. L'Action française, et tout particulièrement la génération Agathon (Massis et Tarde), s'opposent au positivisme et aux orientations de l'État laïque telles qu'elles se manifestent à l'École Normale supérieure (sociologie de Durkheim...). Il ne peut qu'approuver l'opposition à la Troisième République. Mais il

\footnotetext{
${ }^{45}$ Ibid.

${ }^{46}$ Ibid., p. 17.
} 
préfère tous ceux de la génération suivante. Péguy parce qu'il était d'âge mobilisable et qu'il a détruit son génie sans précaution ; d'Annunzio qui est un beau militaire. Je voudrais pouvoir citer Romain Rolland qui a failli avoir une belle attitude, mais qui manque trop de force dans ses gestes et dans ses écrits. J'ajoute que je crois que nous ne sommes plus du tout sur le même terrain. ${ }^{47}$

Drieu ne sait pas dire s'il trouve Barrès antipathique ou sympathique, mais il exprime son « sens du respect ${ }^{48}$ ». Néanmoins la résistance à la Troisième République ne peut plus être un nationalisme étriqué comme celui incarné par l'Action française.

La suite du procès n'apparaît pas dans le numéro 20 de Littérature, dont les 24 pages sont pourtant entièrement consacrées au procès. Étant donné la brièveté des propos manquants, il est peu probable que la déposition du témoin Péret soit prévue dans le numéro 21 jamais paru.

Benjamin Péret est littéralement le lieutenant du témoin allemand absent, et il est plus que cela. Il est revêtu d'une capote de soldat français, maculée de boue, portant un masque à gaz, mais parlant allemand, marchant au pas de l'oie.

L'accusateur : - Sie verstehen nicht ? R. - Nein. Ich bin kaputt. (Il se met au garde-à-vous). L'accusateur : - Raus ! (Il se retire au pas de parade)

Le soldat sacrifié devient à nouveau victime de la violence. Puis le public furieux et révolté entonne la Marseillaise comprenant les lignes notoires : "Marchons, marchons/ Qu'un sang impur/ Abreuve nos sillons. ${ }^{49}$ " Les témoins avec accent, et surtout Péret en habit français avec une voix allemande, créent des indices d'étrangeté physique se dérobant à toute appartenance univoque. À travers l'accent et la langue étrangère, le témoin devient une trace de l'autre sans être l'autre. En tant que tel, il ne peut pas mentir. Il n'est, ni soi-même, ni l'autre. Péret devient un lieutenant, il agit « à la place de ». Ce procédé va plus loin que par exemple chez Siegfried, alias Jacques, ce soldat français amnésique devenu allemand dans Siegfried et le Limousin. Dans le roman et la pièce de théâtre de Giraudoux, le transplanté rentre finalement au pays et retrouve l'identité nationale parsemée de quelques propriétés étrangères.

La performance devient, plus que la pièce de théâtre et le texte écrit, le moyen pour dédifférencier. Le groupe avant-gardiste dont l'inspiration est foisonnante y crée des figures et des fonctions tierces déjouant physiquement les différences nationales et culturelles. Or, avec l'apparition de Péret, on s'éloigne aussi du mimétisme des actes violents. Il incarne tout simplement l'indifférence, c'est-à-dire l'absence de différences, de la créature «kaputt».

${ }^{47}$ Ibid., p. 24

${ }^{48}$ Ibid.

49 Texte reconstruit d'après les épreuves inédites trouvées et publiées par Michel SAnouillet, Dada à Paris (1965), Paris, Flammarion, 1993, p. 272.

Voir aussi Marguerite BONNET, L'affaire Barrès, Paris, José Corti, 1987. 
Il est un enfant sacrifié. Il est témoin d'une façon particulière : tandis que Romoff, Tzara et les autres témoins mènent un débat sur les attitudes intellectuelles à prendre, Péret témoigne de la vérité cachée de la guerre. Il ne témoigne ni pour, ni contre Barrès. Il actualise le tort, l'injustice et la violence perpétrés à l'encontre des hommes, reniés et abusés dans l'héroïsme de la nouvelle culture mémorielle.

Le soldat messager ne peut pas transmettre son message. Le témoin est victime, et de la justice avant-gardiste ( raus »), et de la populace. Le public scandalisé rétablit la binarité et ainsi la réalité porteuse de guerres. Mais ce n'est pas uniquement le bourgeois qui fait disparaître l'oralité et la mise en scène choquantes. Le numéro 20 de la revue Littérature ne donne qu'une version mutilée du procès. Péret, le témoin ainsi que la langue allemande sont évincés. Le texte écrit et publié devient un autre mutilé de guerre. En omettant l'apparition de Péret, la transmission écrite supprime la révolte et le scandale de la violence qui anéantit toute nationalité.

L'historiographie des avant-gardes place précisément ici la césure entre le dadaïsme indifférentiste et le jugement de valeur des futurs surréalistes. Le procès Barrès ferait de façon implicite également le procès des dadaïstes. Or il n'en est rien. Si rétrospectivement la présence des futurs fascistes Ungaretti et Drieu peut déconcerter, Romoff, Tzara et Péret déconstruisent, chacun à sa façon, la dualité belligène par une autre violence. Romoff défend le point de vue révolutionnaire. Tzara rejette violemment l'autorité et détermine les similitudes reliant entre eux les différents « juges ». Péret incarne la créature sacrifiée. Certes, le procès Barrès sonne le glas du dadaïsme. Les champs idéologiques et nationaux conflictuels réapparaissent. Les instances antagonistes du tribunal occupent la position plus politique que Grosz ou Heartfield occupent dans le contexte allemand. Mais entre eux se manifestent les tiers au-delà de ces clivages. Ils témoignent d'un savoir apte à casser les cadres culturels.

L'observateur assiste à la relève d'un mouvement international, le dada, par un mouvement plus mono-culturel, plus français, bien que Max Ernst, Hans Bellmer et Hans-Jean Arp en fassent partie. Il participe aussi à un procès dont les témoins dépassent les dualités.

\section{Conclusion}

Riposter en affirmant le contraire rend impossible une transformation radicale. La négation lie les énergies du contradicteur. Or il est douteux que le comportement agonistique des dadaïstes échappe vraiment aux pièges des oppositions, des objections. L'indifférentisme avec son ni-ni, son ni bellicisme ni pacifisme, prend parfois le chemin d'une impasse, voire l'allure d'un sectarisme. Sur ce point, Tucholsky voit juste. La mobilisation générale du temps défigure aussi les avant-gardes. La formule indifférentiste garde néanmoins son intérêt dans la mesure où elle dépasse la coincidentia 
oppositorum. Elle trouve une application dans les performances des dadaïstes transculturels. Trois figures du tiers - le dandy transfrontalier, l'étranger ni français ni allemand, le témoin lieutenant franco-allemand - provoquent un scandale.

Cependant toute performance, et avec elle toute avant-garde, est passagère. Les lieutenants laissent la place aux représentants. Aujourd'hui les acteurs des cérémonies ont certes changé. Angela Merkel et Nicolas Sarkozy ont déposé ensemble une gerbe sur la tombe du soldat inconnu. Mais, ce faisant, ils ont encore une fois incarné leur pays. Faut-il conclure qu'ils ont encore une fois renié les témoins, les enfants innocents sacrifiés indépendamment de leur nationalité ? 